\title{
Power Prediction Research of Wind Farm Based on LS-SVM Multi-model Modeling \\ Bei Chen*
}

No.60, Shuanglong Street, Qinhuai District, Nanjing City, China

Keywords: LS-SVM, Multi-model, Affinity Propagation Clustering, Wind Power Prediction.

\begin{abstract}
Characteristics of multiple working conditions are existed in the medium-term power prediction model of wind farm. To solve this problem, amulti-model modeling method for power prediction based on LS-SVM algorithm is presented. In the method, affinity propagation clustering algorithm is used to cluster the training samples. Then, the sub-models are trained by LS-SVM. The predicted values of the testing samples are forecasted by the sub-models after being classified by the similarity measurement. Finally, experiments of modeling and prediction are arranged by using the wind farm field data. The experiments show that the proposed method has high prediction accuracy and good generalization ability.
\end{abstract}

\section{Introduction}

The medium-term power prediction system of wind farm is very important. It is usually used for making plans. The wind resource location four country is usually far away from the power center, and this has lead to a serious wind abandon ment. In the process of cross-regional electricity trade to solve large-scale wind power consumption, power prediction of wind farm is an important step. Modeling is the core of the power prediction system. Being affected by the complex terrain and meteorological conditions, characteristics of nonlinear and multi-mode are existed in the modeling of wind power prediction. Single model usually leads to a lower accuracy and a poorer adapt ability.

For the nonlinear and multi-mode problems, a multi-model modeling method is proposed[1].In this method, the overall samples are classified by the clustering algorithm firstly, and then the sub-samples are used to modeling respectively. In this way, the sub-models are trained. The final output of the prediction is the weighted average of the values, which is predicted by the sub-models. The premise of this weighted output method is that the final predicted result is supposed to be a linear combination of the various sub-models. But, in the actual situation, for complex objects, this assumption is not necessarily true[2]. Another multi-model modeling method is based on "switching" method. In this method, The mutual influence between sub-models is eliminated. So it can overcome nonlinear problem and get better adaptability.

Clustering and sub-model modeling are the most two important parts of the multi-model modeling. Affinity Propagation Clustering[4](AP), comparing with the traditional clustering methods, Such as k-means, fuzzy C-means, has higher independence. It can get the clustering center automatically. And it also can reduce the subjective defects brought by human. Least Squares Support Vector Machine[3](LS-SVM) has the advantages of high precision of regression and strong adaptability. It has been widely used in short-term power prediction. And it can also show advantage in medium-term for cast.

According to the above, to solve the medium-term power prediction problem, a multi-model modeling method based on LS-SVM is proposed in this paper. AP and "switching" method are applied to the multi-model modeling. Experiments are carried out by using the samples of wind farm, and the results of the experiments proved that this multi-model modeling method has higher precision and better generalization performance. 


\section{Power Prediction Method in Wind Farm}

\section{Power Prediction of Wind Farm Has A Variety of Methods.}

According to the property of time, it can be divided in toultra-short-term, short-term, medium-term and long-term prediction. Ultra-short-term and short-term prediction is usually for casting within several hours. medium-term prediction is usually for casting within several days. And long-term prediction is usually for casting more than several weeks or months[5].

According to the property of model in galgrithom, power prediction methods of wind farm can be divided into physical method, statistical method, learning method.

The physical method base on the predicted results of the numerical weather prediction (NWP) system, such as the wind speed and wind direction. According to these information and the standard wind power curve, the predicted power of the wind turbines is obtained.

The statistical method does not consider the physical processes, such as wind speed and other weather conditions, it can find the relationship between weather and power based on the historical data. It does the power prediction by using the measured data and numerical weather forecast data of the wind farm[6].

Learning method predicts the wind power mainly throug modeling the nonlinear model base on artificial intelligence algorithm. At present, power prediction method based on learning method mainly include neural network [7], wavelet analysis [8], support vector machine[9], etc.

Based on the learning algrithom, the modeling accuracy is improved effectively. However, in the case of the multiple working conditions of the object, the single model usually suffer from over-learning, local optimal of the iterative process, poor generalization performance, etc. To solve this problems, some scholars started to research multi-modeling method[10]. A method of "switch" multi-model method is proposed, and it can eliminate the interference of sub-model mutual.

\section{Multi-model Modeling Based on LS-SVM}

The multi-model modeling algorithm based on LS-SVM is shown in Fig.1.

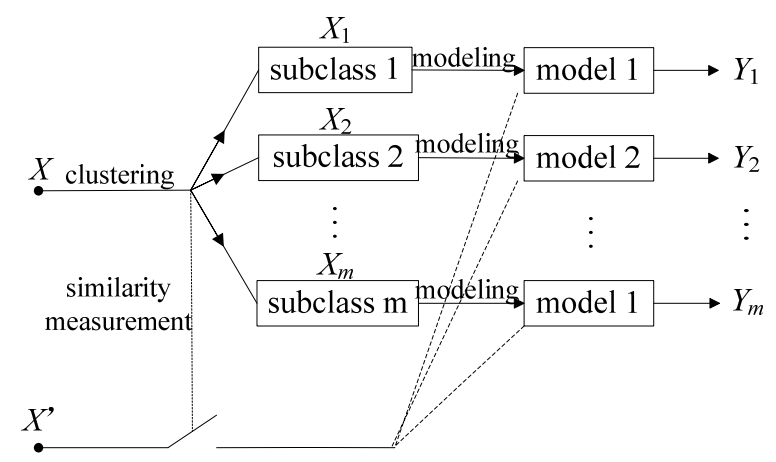

Fig. 1 multi-model modeling and prediction base on "switch" method

First, affinity propagation clustering algorithm is used to cluster the training samples X. And X can be divided into several clusters. Then, LS-SVM is used for sub-model modeling. For every test sample x', it will be classified to one sub-model based on similarity measurement firstly, and then the output y' of the multi-model will be predicted by the sub-model which x' belongs to.

Affinity Propagation Clustering. Affinity propagation clustering was firstly presented in the journal of Science in the year of 2007. It is a fast and efficient clustering method. It does not need to set the cluster centers and the number of the clusters before clustering. It is that each sample competes to be the cluster center by iteration, and then an optimal result will emerge. Because of there duced human intervention, affinity propagation clustering can achieve a better clustering result based on its own situation. This method can solve poor clustering accuracy problem in the multi-model modeling. 
The iterative process of the affinity propagation clustering algorithm is to find the cluster center based on the similarity computation. Set a sample space with n-dimensional. This algorithm is to caculate the adaptability of every sample, and find the optimal one by iteration. After cluster center is found, every sample will caculate the distance between itself and the cluser center. According to this distance, every sample is clustered. The caculation of the distance is based on Euclidean distance.

Sub-model Modeling Based on LS-SVM. Support Vector Machines (SVM)[11] is a learning theory with small samples, based on structural risk minimization principle, which has a better generalization ability. LS_SVM[12] is the improvement of SVM. Compared with the standard support vector machines, LS-SVM avoids solving nonlinear programming problems, reducing computational complexity, accelerating the solving speed. The modeling method based on LS-SVM can solve the problem of small sample and complexity of industrial model. The LS-SVM for regression is presented as follows:

Set examples $\{(x i, y i) \mid i=1, \ldots, N\}$, Here yi $\in R$, and $x i \in R d$ are vectors of $d$ features $x i(n)$, $\mathrm{xi}(\mathrm{n}-1), \ldots, \mathrm{xi}(\mathrm{n}-\mathrm{d}+1)$. The input vector space $\mathrm{Rd}$ is mapped to a high dimensional feature space $\mathrm{Z}$ based on kernel function $\varphi(x i)$. By this way, the nonlinear problem is transformed into linear question, as follow formula (1), which is in the high dimensional space $\mathrm{Z}$.

$$
y(x)=w^{T} \varphi(x)+b, w \in Z, b \in P
$$

Here $\mathrm{w}$ and $\mathrm{b}$ is parameter to be identified. The optimization problem is defined as

$$
\min J(w, e)=0.5 w^{T} w+0.5 \gamma \sum_{i=1}^{N} e_{i}^{2}, \gamma>0
$$

Equality constraints is

$$
y_{i}=w^{T} \varphi\left(x_{i}\right)+b+e_{i}, i=1,2, \ldots, N
$$

Where the first part of the objective function (2) corresponds to the generalization ability of the model, and the second part corresponds to the accuracy. The parameter of $\gamma$ is a balance of generalization capability and precision, which can be adjusted manually .ei is the difference between actual output and predicted output in training.

Solving the optimization problem by exploiting the Lagrange multipliers approach, the optimal solution is

$$
\left[\begin{array}{l}
\mathrm{b} \\
\alpha
\end{array}\right]=\left[\begin{array}{cc}
0 & \overrightarrow{1}^{\mathrm{T}} \\
\overrightarrow{1} & \Omega+\gamma^{-1} \mathrm{I}
\end{array}\right]^{-1}\left[\begin{array}{l}
0 \\
\mathrm{y}
\end{array}\right]
$$

Where $\mathrm{y}=\left[\begin{array}{llll}\mathrm{y} 1 & \mathrm{y} 2 & \ldots & \mathrm{yN}\end{array}\right] \mathrm{T}, \overrightarrow{1}=\left[\begin{array}{llll}1 & 1 & \ldots & 1\end{array}\right] \mathrm{T}, \alpha=\left[\begin{array}{llll}\alpha 1 & \alpha 2 & \ldots & \alpha \mathrm{N}\end{array}\right] \mathrm{T}, \Omega$ is a symmetric matrix with the dimensional of $\mathrm{N} \times \mathrm{N} . \Omega \mathrm{ij}=\varphi(\mathrm{xi}) \mathrm{T} \varphi(\mathrm{xj})=\mathrm{K}(\mathrm{xi}, \mathrm{xj}), \mathrm{i}, \mathrm{j}=1,2, \ldots, \mathrm{N}, \mathrm{K}$ is the kernel function. As a result, the estimating function $\mathrm{y}$ can be expressed as

$$
y(x)=\sum_{i=1}^{N} \alpha_{i} K\left(x, x_{i}\right)+b
$$

Multi-model Modeling and Forecast. The steps of multi-model modeling base on LS-SVM and the prediction are as follows:

First, the training samples clustering based on AP. First of all, samples normalized. Each sample is normalized to -1 to 1 , this is to eliminate dimensionless impact. Then the initialization of $\lambda$ and $p$. $\lambda$ can be set at 0.5 to 0.9 according to the size of the samples. $p$ can be set as $p m=$ median(S).

To improve the clustering quality, the index of Silhouette[13] is introduced. Silhouette reflects the tightness of samples which from the same class and the divisibility of samples which from 
different classes. We call the index Sil_av in this paper. p will be adjusted based on Sil_av. The setting step is as

$$
\mathrm{p}_{\text {step }}=\frac{0.01 \mathrm{p}_{\mathrm{m}}}{0.1 \sqrt{\mathrm{k}+50}}
$$

Where $\mathrm{k}$ is the center of cluster. Choose a value of $\mathrm{p}$ to cluster the sample who makes Sil_avto be the biggest.

Second, sub-model training. Sub-model modeling based on LS-SVM. RBF kernel function is used to be the kernel function.

$$
K\left(x, x_{i}\right)=e^{\left\{\frac{-\left\|x-x_{i}\right\|^{2}}{\sigma^{2}}\right\}}
$$

The $\gamma$ and $\sigma$ of the LS-SVM are optimized based on cross validation, and the objective function is defined as

$$
\text { PRESS }=\sum_{\mathrm{i}}\left(\mathrm{y}_{\mathrm{i}}-\mathrm{y}_{-\mathrm{i}}\right)^{2}
$$

Where yi is the measured value, $y-i$ is the predicted value of the model which is trained by the samples except xi. The smaller the press is, the better the regression accuracy is, and more suitable the $\gamma$ and $\sigma$ is.

At last, multi-model prediction and output. For every test sample xt, by calculating the similarity of $\mathrm{xt}$ and the center of every sub-cluster, will be classified to the cluster $\mathrm{Cn}$ which has the biggest similarity. Then the forecast of $\mathrm{xt}$ is done by using the sub-model which is trained by cluster $\mathrm{Cn}$. In this paper, Euclidean distance is used to calculate the similarity.

\section{Experimentation and Analysis}

We choose a wind farm which has a total number of 158 wind turbines with the brand of GAMESA. We call the wind turbine unit in this paper. Of the 158 units, 58 were model of G52-850, and the capacity of them is $850 \mathrm{~kW} .100$ were model of G90-200, and the capacity of them is $2000 \mathrm{~kW}$. The total capacity of the wind farm is $249,300 \mathrm{~kW}$.

We got samples, within one week, from the database in the wind farm for experiments. Data interval is 15 minute. There are 570 groups of samples remaining after removing invalid data. Of the 570 samples, the first 380 samples were used for training model, and the last 190 samples were used for prediction. We selected the wind speed, temperature, humidity and pressure as the secondary variable, and selected the power of whole wind farm as the output variable. Modeling and prediction experiments were performed as the steps presented above.

First, training samples clustering based on AP. We got a highest Silhouette when the training samples were divided into 6 classes based on adjusting p. At this time, Sil_av $=0.6632$. Six sub-models were trained by samples of the six classes. And the $\gamma$ and $\sigma$ of the LS-SVM are also optimized based on cross validation[1].

Second, sub-model prediction. The every test sample was classified to 6 classes following the 6 sub-models. Euclidean distance is the measurement of classification. Then, the predicted result of every test sample was output by the sub-model.

Last, record the result of experiments. The result of LS-SVM multi-model modeling are as follows:

Fig. 2 shows the predicted value of all 570 samples base on the multi-model modeling method proposed by this paper. The first 380 samples were used for model training, and the 570 samples were used to predicting by using the model trained. It can be seen from Fig. 2 that the overall predicted value of prediction is in good agreement with the actual measured values.

The root mean square error (RMSE) and maximum absolute error (MAXE) were used to evaluate the prediction of the model[13]. 


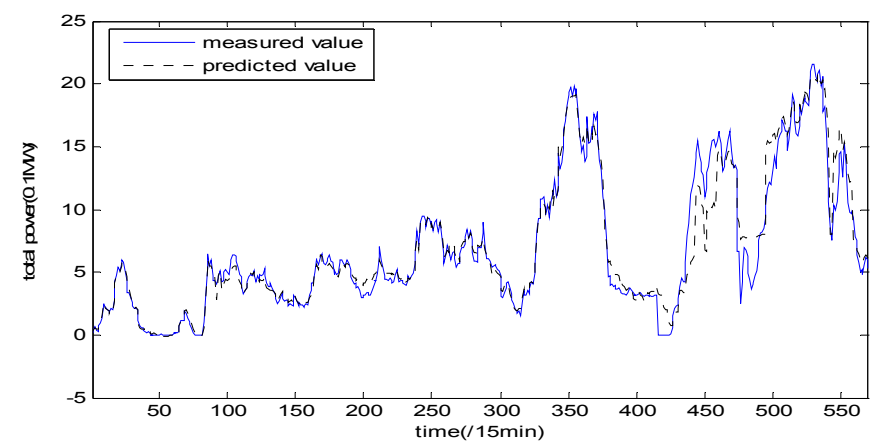

Fig.2 The predicted results of LS-SVM multi-model modeling

$$
\begin{gathered}
R M S E=\sqrt{\frac{1}{n} \sum_{l}^{n}\left(y_{i}-\widehat{y}_{l}\right)^{2}} \\
\text { MAXE }=\max _{1 \leq \mathrm{x} \leq \mathrm{n}}\left(\left|\mathrm{y}_{\mathrm{i}}-\widehat{\mathrm{y}}_{\mathrm{l}}\right|\right)
\end{gathered}
$$

LS-SVM single model modeling and the commonly used interpolation method based on wind power curve were used to carry out experiments base on the same samples and grouping cases. The prediction errors of the three methods, with 190 test samples, are recorded in Table 1.

Table 1prediction errors of modeling methods

\begin{tabular}{|c|c|c|}
\hline Modeling Method & RMSE & MAXE \\
\hline Interpolationfitting & 2.99183 & 8.41214 \\
\hline LS-SVM single model & 2.53732 & 7.24730 \\
\hline LS-SVM multi-model & 2.20565 & 6.20895 \\
\hline
\end{tabular}

In the experiment of interpolation fitting method, we got the result by fitting the standard wind power curve which provided by the manufacturer of GAMESA. In this method, the wind speed is used as input value, and the output of the fitting is the predicted result. In the experiment of LS-SVM single model, single model modeling was done based on the first 380 samples, and prediction was done based on remained 190 samples.

Compared the errors of Interpolation fitting, LS-SVM single mode, LS-SVM multi-model, it can be seen that the regression accuracy of LS-SVM multi-model is better than other modeling methods. The reason is that multiple working conditions are existed in the modeling object, and the multi-model modeling can better adapt to this feature. The adaptability of the sinlge modeling is not good enough to deal with this feature. Only wind speed is considered in the Interpolation fitting method, and the result can only be better in ideal working status. So the modeling accuracy is low in complex situation.

\section{Conclusions}

A multi-model modeling method based on LS-SVM is introduced to solve the nonlinear and multi-condition problems in medium-term power prediction. It is concluded that the method of multi-model modeling based on LS-SVM is feasible and effective in medium-term power prediction base on theoretical analysis and modeling experiments. The samples used in the experiments of this paper are historical data. The next step is to use the actual weather forecast data for prediction experiments. 


\section{References}

[1]Song Kun,LiLijuan,ZhaoYingkai. LS-SVM Multi-model Modeling Method Based on AP[J]. Computer Engineering,2011,37(14): 169-171.

[2]Li Xiuliang,SuHongye,ChuJian. Multi-model Soft Sensor Modeling Based on On-line Clustering and Relevance Vector Machine [J].Control and Instruments in Chemical Industry. 2008, 35 (3):34-37.

[3]GuYanping,ZhaoWenjie,WuZhansong.Least squares support vector machine algorithm[J]. Journal of Tsinghua University(Science and Technology),2010(7): 1063-1066.

[4]Sun Maowei,YangHuizhong.Multi-model soft-sensor modeling based on improved affinity propagation clustering algorithm and application[J]. Journal of Nanjing University of Science and Technology,2016,40(2):72-75.

[5]GaoYang,ChenHua-yu,OU Yang-qun.ASummery of Studies on Wind Power Prediction Technologies[J]. Power System and Clean Energy. 2010,26(4):60-63.

[6]GU Xing-kai,FanGao-feng,Wang Xiao-rong,etc.Summarization of Wind Power Prediction Technology[J]. Power System Technology,2007(S2):335-338.

[7]Li S.Wind Power Prediction Using Recurrent Multilayer Perceptron Neural Networks[A]. in: Power Engineering. Society General Meeting.IEEE[C].2003.2325-2330.

[8]L Cao,R Li.Short-term wind speed prediction model for wind farm based on wavelet decomposition [J]. IEEE Trans on DRPT,2008:2525-2529.

[9]QiuJie,PeiRuiping,ZhangAnchuan,Li Kan.Chaotic and LS-SVM for short-term wind power forecast[J]. Journal of Chongqing Technology and Business University(Natural Science Edition),2015, 32(3):47-50.

[10]WANG Li-jie,DONGLei,HUGuo-fei,etc.Combine prediction of wind power generation in multi-dimension embedding phase space[J]. Control and decision,2010(4):577-580.

[11]Li Lijuan,Su Hongye,Chu Jian. Modeling of Isomerization of C8 Aromatics by Online Least Squares Support Vector Machine. Chinese Journal of Chemical Engineering, 2009, 17(3):437-444.

[12] LijuanLi.The Study of Modeling Algorithm Based on LS-SVM and Predictive Control Algorithm, ZheJiang University PhD thesis,2008.

[13]WANG Kai-Jun,ZHANG Jun-Ying,LiDan,etc.Adaptive Affinity Propagation Clustering[J].ActaAutomatica Sinica,2007,33(12):1242-1246.

[14]MU Chao-xu, LIANGRui-xin, LIXun-ming. Allied Inverse Controller for LSSVM in Nonlinear Systems[J]. Computer Engineering, 2009,35(12):181-183.

[15]Yang Hui,Chai Tianyou. Component Content Soft-sensor Based on Neural Networks in Rare-earth Countercurrent Extraction Process[J].ActaAutomatica Sinica,2006,32(4): 489-495. 